\title{
RESTRICTIONS OF MODULES TO LOCAL SUBGROUPS
}

\author{
DAVID W. BURRY ${ }^{1}$ AND JON F. CARLSON ${ }^{2}$
}

\begin{abstract}
The main theorem of this paper is a strengthening of the uniqueness statement in the Green correspondence for modules over certain group rings of finite groups. One consequence of this result is an extension of the work of J. L. Alperin and the first author on a module-theoretic approach to block theory. Specifically, the stronger Green correspondence is used to complete the proof of Brauer's First Main Theorem.
\end{abstract}

1. Introduction. Let $p$ be a prime and let $P$ be a $p$-subgroup of a finite group $G$. Suppose that $H$ is a subgroup of $G$ with $N_{G}(P) \subseteq H$. Under the usual Green correspondence, each indecomposable $H$-module $U$, with vertex $P$, is related to a unique indecomposable $G$-module $V$ which has the property that $U$ is a component of $V_{H}$ (the restriction of $V$ to an $H$-module) and which also has vertex $P$. The main result of this paper is that the condition that $V$ have vertex $P$ is unnecessary. That is, if an indecomposable component $U$ of $V_{H}$ has vertex $P$, then $V$ must also have vertex $P$, and $U$ and $V$ must be Green correspondents. This theorem when combined with the results in [1] yields a module-oriented proof of Brauer's First Main Theorem of Blocks.

The proof of the main theorem (Theorem 5) has two major steps. The first is to establish, in $\S 2$, an onto condition for certain trace maps. This was proved in a more general context by Green [4], but we include a proof for the sake of completeness. The second step uses well-known facts about the structure of the endomorphism rings of indecomposable modules. The procedure is similar to that which was employed by Scott in [5]. In fact, Theorem 5 can be viewed as a generalization of Theorem 3(b) of [5]. We would like to thank J. L. Alperin for directing our attention to Scott's result.

2. Trace functions. Let $p$ be a prime integer. Let $R$ be a suitable $p$-coefficient ring such as a field of characteristic $p$ or a finite extension of the $p$-adic integers (see Hypothesis 52.1 of [3]). We consider only finite groups in this paper. If $G$ is a group, then a $G$-module is a finitely generated $R$-free right $R G$-module. The reader is referred to [2] or [3] for the definitions of such terms as "vertex", "relatively projective" and "Green correspondence". A component of a $G$-module is an indecomposable direct summand of that module.

Received by the editors April 28, 1980.

1980 Mathematics Subject Classification. Primary 20 C20.

Key words and phrases. Group rings, block theory, Green correspondence, vertex of a module.

'Supported in part by NSF Grant MCS 7904773.

${ }^{2}$ Supported in part by NSF Grant MCS 7801685. 
Suppose that $H$ is a subgroup of $G$ and that $V$ is a $G$-module. Let $V_{H}$ denote the restriction of $V$ to an $H$-module and let $E_{H}(V)=\operatorname{Hom}_{R H}\left(V_{H}, V_{H}\right)$. If $g \in G$, $\varphi \in E_{H}(V)$, then define $\varphi^{g} \in E_{H^{8}}(V)$ to be the homomorphism given by $(v) \varphi^{g}=$ $\left(v g^{-1}\right) \varphi \cdot g$ for all $v \in V$. Here $H^{g}=g^{-1} H g$. The trace function $\operatorname{Tr}_{H, G}: E_{H}(V) \rightarrow$ $E_{G}(V)$ is defined for $\varphi \in E_{H}(V)$ by $\operatorname{Tr}_{H, G}(\varphi)=\sum \varphi^{g}$ where the sum is taken over a complete set of representatives of the right cosets of $H$ in $G$. The trace function is independent of the choice of representatives. It is an $R$-module homomorphism, but it is not a ring homomorphism. However, it is easy to verify that it has the following property.

LeMma 1. If $\varphi \in E_{H}(V)$ and $\psi \in E_{G}(V)$, then $\psi \circ \operatorname{Tr}_{H, G}(\varphi)=\operatorname{Tr}_{H, G}(\psi \circ \varphi)$ and $\operatorname{Tr}_{H, G}(\varphi) \circ \psi=\operatorname{Tr}_{H, G}(\varphi \circ \psi)$. In particular the set $E_{H, G}(V)=\operatorname{Tr}_{H, G}\left(E_{H}(V)\right)$ is a two-sided ideal in $E_{G}(V)$.

The definition of $\operatorname{Tr}_{H, G}$ is motivated by the criterion of Higman which states that a $G$-module $V$ is relatively $H$-projective if and only if the identity homomorphism, $\operatorname{Id}_{V}$, is in $E_{H, G}(V)$ (see Lemma 51.2 of [3]). Using Lemma 1 we may prove the following.

LEMMA 2 (ROSENBERG's LeMMA). Let $S$ be a set of subgroups of $G$ and let $V$ be an indecomposable $G$-module. If $\operatorname{Id}_{V} \in \Sigma_{H \in S} E_{H, G}(V)$ then $V$ is relatively $H$-projective for some $H \in S$.

Proof. Suppose that there exist elements $\varphi_{H} \in E_{H}(V)$ such that $\operatorname{Id}_{V}=$ $\sum_{H \in S} \operatorname{Tr}_{H, G}\left(\varphi_{H}\right)$. Since $V$ is indecomposable we know that $E_{G}(V)$ is a local ring (see Theorem 43.3 of [3]). Hence for some $H \in S, \operatorname{Tr}_{H, G}\left(\varphi_{H}\right) \in E_{H, G}(V)$ is invertible in $E_{G}(V)$.

If $H$ is a subgroup of $G$ and if $V$ is a $G$-module, then let $\operatorname{Res}_{G, H}: E_{G}(V) \rightarrow$ $E_{H}(V)$ denote the restriction homomorphism. It is in fact the inclusion $E_{G}(V) \subseteq$ $E_{H}(V)$. This is always an $R$-algebra monomorphism.

Lemma 3 (Mackey Decomposition). Let $P$ and $H$ be subgroups of $G$. Let $V$ be a $G$-module and suppose that $\lambda \in E_{P}(V)$. Then

$$
\operatorname{Res}_{G, H}\left(\operatorname{Tr}_{P, G}(\lambda)\right)=\sum_{t} \operatorname{Tr}_{P^{t} \cap H, H}\left(\operatorname{Res}_{P^{t}, P^{t} \cap H}\left(\lambda^{t}\right)\right),
$$

where the sum is over a complete set of representatives of the $(P, H)$-double cosets in G.

Proof. It is easy to see that if $\left\{h_{1}, \ldots, h_{s}\right\}$ is a complete set of representatives of the right cosets of $P^{t} \cap H$ in $H$, then $\left\{t h_{1}, \ldots, t h_{s}\right\}$ is a complete set of representatives of the right cosets of $P$ in $P t H$. The lemma now follows from the observation that

$$
\sum_{i=1}^{s} \lambda^{t h_{i}}=\operatorname{Tr}_{P^{t} \cap H, H}\left(\operatorname{Res}_{P^{t}, P^{t} \cap H}\left(\lambda^{t}\right)\right) \text {. }
$$

The following, which is the principal result of this section, is a special case of Theorem 2(i) of [4]. It is a direct consequence of Lemma 3 and the fact that $\operatorname{Tr}_{H, G} \circ \operatorname{Tr}_{P, H}=\operatorname{Tr}_{P, G}$. 
THEOREM 4. Suppose that $P$ is a p-subgroup of $G$ and that $H$ is a subgroup of $G$ with $N_{G}(P) \subseteq H$. Let $S=\left\{P^{t} \cap H \mid t \notin H\right\}$. For any $G$-module $V$ let $J=$ $\Sigma_{Q \in S} E_{Q, G}(V)$. Then for any $\varphi \in E_{P}(V)$

$$
\operatorname{Res}_{G, H}\left(\operatorname{Tr}_{P, G}(\varphi)\right) \equiv \operatorname{Tr}_{P, H}(\varphi) \text { modulo } J .
$$

If $r: E_{P, G}(V) \rightarrow\left(E_{P, H}(V)+J\right) / J$ is the composition of $\operatorname{Res}_{G, H}$ and the natural quotient modulo $J$, then $r$ is an algebra epimorphism.

3. The main theorem. The above theory is now applied to obtain a new result.

THEOREM 5. Let $P$ be a p-subgroup of a group $G$. Let $H$ be a subgroup of $G$ with $N_{G}(P) \subseteq H$. If $V$ is an indecomposable $G$-module and if $V_{H}$ has a component $U$ with vertex $P$, then $V$ has vertex $P$ and $V$ is the Green correspondent of $U$.

Proof. Let $J, r$ be as in Theorem 4. Clearly $r$ extends to a ring homomorphism

$$
\hat{r}: E_{G}(V) \rightarrow\left(E_{H}(V)+J\right) / J
$$

which is defined in the same fashion. Let $e \in E_{H}(V)$ be the primitive idempotent that is a projection onto $U$. Now $e \in E_{P, H}(V)$, but by Lemma 2, $e \notin J$. Theorem 4 implies that there exists $u \in E_{P, G}(V)$ such that $r(u)=e+J$. Since $V$ is indecomposable we have that $E_{G}(V)$ and $E_{G}(V) / \operatorname{ker} \hat{r}$ are local rings. But since $u+\operatorname{ker} \hat{r}$ is a nonzero idempotent in $E_{G}(V) / \operatorname{ker} \hat{r}$, it must be invertible. So $u$ is invertible in $E_{G}(V)$, and we have that $E_{G}(V) \subseteq E_{G}(V) \cdot u \subseteq E_{P, G}(V)$ since $E_{P, G}(V)$ is an ideal. This implies that $\operatorname{Id}_{V} \in E_{P, G}(V)$ and that $V$ has vertex $P$.

Another useful form of Theorem 5 is the following. Let $V$ be an indecomposable $G$-module. For a $G$-module $X$ write $X=X_{1} \oplus \cdots \oplus X_{t}$ where each $X_{i}$ is indecomposable, the multiplicity of $V$ in $X$ is the number of the components $X_{i}$ which are isomorphic to $V$.

THEOREM 6. Let $P$ be a p-subgroup of $G$, and let $H$ be a subgroup of $G$ with $N_{G}(P) \subseteq H$. Let $U$ be an indecomposable $H$-module with vertex $P$, and suppose that $V$ is an indecomposable $G$-module which is the Green correspondent of $U$. For any $G$-module $X$, the multiplicity of $V$ in $X$ is the same as the multiplicity of $U$ in $X_{H}$. Moreover the number of components with vertex $P$ in any decomposition of $X$ is the same as the number of components with vertex $P$ in any decomposition of $X_{H}$.

4. An application to the module-oriented theory of blocks. The article [1] presents a treatment of block theory that relies solely on the general properties of modules for group rings. The program is satisfactorily complete except for a proof of Brauer's First Main Theorem. Fix a $p$-subgroup $P$ of $G$ and let $H$ be a subgroup of $G$ with $N_{G}(P) \subseteq H$. The authors in [1] were only able to establish that the Brauer correspondence gives a bijection between the set of blocks of $G$ with defect group $P$ and a subset of the blocks of $H$ with defect group $P$ (see Theorem 3.6(2)). What has been missing is a general module-theoretic result which shows that $G$ and $H$ have the same number of blocks with defect group $P$. Relying on the results and notation of [1] we can easily establish this missing fact using Theorem 6. The blocks of $G$ with defect group $P$ are by definition the components of the $(G \times G)$ module $R G$ which have vertex $\delta(P)$. By Theorem 6, $R G$ and $(R G)_{H \times H}$ have the same number of components with vertex $\delta(P)$. Now Lemma 3.3 of [1] states that 
there is a decomposition $(R G)_{H \times H} \cong R H \oplus M$ where none of the components in $M$ have vertex $\delta(P)$. Hence relative to this decomposition, any component of $(R G)_{H \times H}$ which has vertex $\delta(P)$ must lie in $K H$. Such components are, by definition, the blocks of $H$ with defect group $P$.

\section{REFERENCES}

1. J. L. Alperin and D. W. Burry, Block theory with modules, J. Algebra 65 (1980), 225-233.

2. D. W. Burry, A strengthened theory of vertices and sources, J. Algebra 59 (1979), 330-344.

3. L. Dornhoff, Group representation theory, Dekker, New York, 1971.

4. J. A. Green, Axiomatic representation theory for finite groups, J. Pure Appl. Algebra 1 (1971), 41-77.

5. L. L. Scott, Modular permutation representations, Trans. Amer. Math. Soc. 175 (1973), 101-121.

Department of Mathematics, Yale University, New Haven, Connecticut 06520

Department of Mathematics, University of Georgia, Athens, Georgia 30602 (Current address of Jon F. Carlson)

Current address (David Burry): Department of Mathematics and Physics, University of Hartford, West Hartford, Connecticut 06117 